\title{
YouTube as a source of information: How good is the quality and reliability of videos related to obsessive compulsive disorder?
}

\author{
Hasan Kaya ${ }^{1 \oplus}$, Alper Bulbul $^{(\oplus}$, Aybeniz Civan Kahve $^{1 \odot}$ \\ ${ }^{1}$ University of Health Sciences, Ankara City Hospital, Department of Psychiatry, Ankara, Turkey \\ ${ }^{2}$ University of Health Sciences, Erenkoy Training and Research Hospital for Psychiatry and Neurological Diseases, Department of \\ Psychiatry, Istanbul, Turkey
}

\begin{abstract}
Objective: YouTube (Google LLC, Mountain View, CA, USA), an online video-sharing platform, has become a resource for health information. However, the quality of the information presented to viewers is not well known. This study is an examination of quantitative and qualitative characteristics of YouTube videos related to obsessive-compulsive disorder (OCD) and provides analysis of data of the number of views and the relationship between the popularity and quality of videos related to OCD.

Method: This was a qualitative, cross-sectional study. A total of 131 English-language videos related to OCD available on YouTube were reviewed. The DISCERN questionnaire, the Global Quality Scale (GQS), and a YouTube OCD-Specific Score instrument were used to evaluate the videos. Details of the length of the videos, the number of views, the number of likes/ dislikes, and the length of time since upload were analyzed.

Results: The source of the videos was most often a psychiatrist/psychologist ( $n=63,48.1 \%$ ), followed by an educational course $(n=21,16 \%)$, and patients/relatives $(n=16,12.2 \%)$. Most of the videos were of low quality. The DISCERN instrument ratings indicated that only $36.6 \%$ of the videos were of fair quality, and GQS assessment revealed that only $27.5 \%$ of videos scored $\geq 3$ points.

Conclusion: The OCD-related video content reviewed was largely inadequate. Information on the disease and distinction between symptoms, as well as treatment options, was insufficient. YouTube is a widely used information resource. Greater participation of mental health professionals could help to ensure access to high quality information on OCD, which could be of great benefit to patients and the public.
\end{abstract}

Keywords: Internet, mental illness, obsessive-compulsive disorder, YouTube

\section{INTRODUCTION}

The internet has become an increasingly important source of information on various topics, including health. Some $70 \%$ of internet users first turn to online sources when they have a health-related question (1).
While the availability of health information on the internet offers several advantages, it is evident that there are also potential dangers. The average user may have difficulty making an appropriate evaluation of the quality of the available medical information (2). Internet users generally have high confidence in

How to cite this article: Kaya H, Bulbul A, Kahve AC. YouTube as a source of information: How good is the quality and reliability of videos related to obsessive compulsive disorder? Dusunen Adam The Journal of Psychiatry and Neurological Sciences 2021;34:368-374.

Correspondence: Hasan Kaya, University of Health Sciences, Ankara City Hospital, Department of Psychiatry, Ankara, Turkey 
information found online (3), however, many sites contain incorrect or incomplete information (4).

YouTube (Google LLC., Mountain View, CA, USA) is currently the largest online video-sharing platform, with more than 2 billion users. Viewers across the globe now watch more than 1 billion hours of videos a day (5). It is the second most used internet site worldwide (6). It has become an information platform in addition to providing entertainment. YouTube now includes an ever-growing health-related video archive for medical students, doctors, patients, and relatives $(7,8)$.

Obsessive-compulsive disorder (OCD) has an estimated lifetime prevalence of $1.5 \%$ to $3.5 \%$ in men and women $(9,10)$. It is a chronic and disabling disease that negatively affects patients' academic, professional, social, and family functioning (11). OCD is often described as a hidden disorder (12). The disease has an egodystonic nature and patients may feel embarrassed and hide their symptoms. As a result, they may seek help less often and the complexities of the disorder may be misunderstood (13). Diagnosed or undiagnosed OCD patients and their relatives may search for information about the disease online as a result of the burden of the illness and possible shame related to seeking help elsewhere. It has been demonstrated that patients receiving treatment for psychiatric disorders often use the internet to search for general and medical information (14). Notably, individuals with OCD frequently use the internet as a source of information (15).

The number of health-related videos on YouTube has increased in recent years. The growing use and reach of this platform indicate that the quality and reliability of the content are even more critical and this issue has become an important subject of research.

This study was designed to examine the content of YouTube videos related to OCD to assess the information provided about the disease. Data of the number of views and the relationship between a video's popularity and quality were also evaluated. To the best of our knowledge, this is the first published research to evaluate OCD videos presented on YouTube. A qualitative assessment of the information presented could represent a valuable contribution to the literature and recommendations related to health information provided online.

\section{METHOD}

This study did not require ethical approval since it only involved public access data.

\section{Search Strategy}

A search of the YouTube website (http://www.youtube. com) was performed using the keyword "obsessivecompulsive disorder" on December 20, 2020. A previously unused browser and the standard YouTube filters were used to retrieve videos according to their relevance.

As users typically do not look at more than the first 5 pages of search results, other studies have used the first 5 pages of each keyword search (20 videos/page $\times 5$ pages $=100$ videos $)(16,17)$. However, to provide a broad evaluation and greater accuracy, in this study, a total of 250 videos in the first 13 pages were reviewed. A document was created to save the uniform resource locator (URL) and header of the 250 videos reviewed.

The following types of videos were excluded: nonEnglish $(n=106)$, without sound $(n=3)$, less than a minute in length $(\mathrm{n}=2)$, related to obsessive-compulsive personality disorder $(n=7)$, duplicate $(n=1)$. A total of 131 videos were evaluated. Two experienced psychiatrists $(\mathrm{AB}, \mathrm{ACK})$ independently reviewed all of the videos to increase reliability. Each of the psychiatrists evaluated and scored the videos. Statistical comparisons were made using the DISCERN questionnaire, the Global Quality Scale (GQS), and a YouTube OCD-Specific Score (YOCD-SS) developed by the authors. The inter-rater reliability was examined, and the statistical analysis was performed using the average of the scores.

\section{Video Evaluation}

The length (minutes) of the videos, the number of views, the number of likes and dislikes, and the length of time since the upload (days) were recorded. A view ratio formula $\left(\frac{\text { number of views }}{\text { the time since upload (days), }}\right)$ was used to evaluate the popularity of the videos, as well as a like ratio (like+dislike $x 100)$ and a video power index (VPI: $\left.\frac{\text { like ratio } x \text { view ratio }}{100}\right)(17,18)$. The quality of information was assessed with 3 measurement tools.

\section{DISCERN Instrument}

The DISCERN questionnaire was created as a means to assist consumers of health information with an assessment of the quality of written information about treatment options. It consists of a total of 16 questions; a higher score indicates better quality. The first part consists of 8 questions about reliability of the source, the second part has 7 questions that focus on specific details of the treatment choices presented. The final question is a general quality rating of the publication. Each item is scored on a 5-point scale. A score of 1 is a 


\section{Table 1: The DISCERN questionnaire (1-5 points for each item)}

\section{Reliability score}

Q1. Are the aims clear?

Q2. Does it achieve its aims?

Q3. Is it relevant?

Q4. Is it clear what sources of information were used to compile the publication (other than the author or producer)?

Q5. Is it clear when the information used or reported in the publication was produced?

Q6. Is it balanced and unbiased?

Q7. Does it provide details of additional sources of support and information?

Q8. Does it refer to areas of uncertainty?

\section{Treatment score}

Q9. Does it describe how each treatment works?

Q10. Does it describe the benefits of each treatment?

Q11. Does it describe the risks of each treatment?

Q12. Does it describe what would happen if no treatment was used?

Q13. Does it describe how treatment choices affect overall quality of life?

Q14. Is it clear that there may be more than one possible treatment choice?

Q15. Does it provide support for shared decision-making?

\section{Overall impression}

Q16. Based on the answers to all of the above questions, rate the overall quality of the publication as a source of information about treatment choices.

response of "definitely no," 2 reflects an answer of "generally no," 3 points are awarded for a publication that "partially" meets the criteria, 4 is a response of "generally yes," and 5 is a judgement of "definitely yes" (19). The DISCERN scale was used to group videos in this study into quality categories of excellent (63-75 points), good (51-62 points), fair (39-50 points), poor (27-38 points), and very poor (16-26 points) (Table 1$)$.

\section{Global Quality Scale}

The GQS was used to evaluate the overall quality of the videos. This instrument assesses the video quality, streaming, and ease of use of the information presented. The scoring system uses a scale of 1 to 5 and includes quality assessment of the content, information coverage, and patient utility (20). One point means poor quality, poor flow of the site, no information, and very unlikely to be of use to patients; 2 points indicate generally poor quality and poor flow, some information but many important topics missing, and of very limited use to patients; 3 points are awarded for a video with moderate quality, suboptimal flow, some important information is adequately discussed, but important topics are missing, and it is judged to be somewhat useful to patients; 4 points mean good quality and generally good flow, the most important topic is discussed, and it is useful to patients; and 5 points indicate excellent quality and excellent flow, the most important topic is covered, and it could be very useful to patients.

\section{YouTube Obsessive-Compulsive Disorder-Specific Score}

The authors developed a YouTube Obsessive Compulsive Disorder-Specific Score instrument, since no similar tool is known to exist. A scoring system was developed based on scales used to determine the quality of YouTube videos related to other diseases $(16,17)$. A total of 13 items are used to make the assessment: Diagnosis (questions 1, 2), epidemiology (questions 3-5), etiology (questions 6, 7), symptoms (questions 8-10), treatment (questions 11, 12), and prognosis (question 13). Each item is given a score between 0 and 2 to indicate the adequacy of the information presented; 0 points indicates that the applicable information was not provided, 1 point indicates insufficient information, and 2 points are awarded for comprehensive information. The quality of the video was scored based on the total score as very poor (0-5), poor (6-10), fair (11-15), good (16-20), or excellent (21-26). The questions used in the scoring system are provided in Table 2 . 
Table 2: The YouTube obsessive-compulsive disorderspecific score instrument (0-2 points for each item)

\section{Diagnosis}

Q1. Psychiatric interviews in the diagnostic evaluation

Q2. Differential diagnosis information

\section{Epidemiology}

Q3. The age range of the disease

Q4. Gender differences

Q5. Knowledge about prevalence

\section{Etiology}

Q6. Biological risk factors

Q7. Psychosocial risk factors

\section{Symptoms}

Q8. Definition of obsession

Q9. Definition of compulsion

Q10. Impairment in functionality

\section{Treatment}

Q11. Pharmacological treatment

Q12. Psychotherapy options

\section{Prognosis}

Q13. Information on the course of the disease (chronicity)

Q: Question.

\section{Statistical Analysis}

IBM SPSS Statistics for Windows, Version 22.0 software (IBM Corp., Armonk, NY, USA) was used to perform the statistical analysis. The KolmogorovSmirnov test was used to assess the conformity of continuous variables to normal distribution. Continuous variables are presented as median \pm interquartile range (IQR) values and categorical variables as numbers and percentages. For variables that did not demonstrate normal distribution, the Mann-Whitney $U$ test was used to compare 2 independent groups. Spearman's correlation was used to analyze the relationship between variables that were not distributed normally. In order to investigate the item reliability of the DISCERN and YOCD-SS, the internal consistency was evaluated using Cronbach's alpha coefficients. A $p$ value of $<0.05$ was considered statistically significant.

\section{RESULTS}

A total of 131 videos were evaluated. The mostwatched video was 3.5 minutes in length, had been available on YouTube for 1649 days, and had been viewed 6,815,630 times, while the least-watched video
Table 3: Descriptive statistics of the YouTube videos reviewed related to obsessive-compulsive disorder

\begin{tabular}{lccc} 
& Median & IQR & Range \\
\hline Number of views & 13745 & 50839 & $44-6815630$ \\
Length, (min) & 5.78 & 8.50 & $1.1-70.70$ \\
Days since upload & 1014 & 1668 & $8-4985$ \\
Number of likes & 187 & 888 & $0-180021$ \\
Number of dislikes & 6 & 22 & $1-2517$ \\
Like ratio & 96.75 & 5.0 & $50-100$ \\
View ratio & 13.85 & 56.45 & $0.11-5751.11$ \\
VPI score & 13.30 & 53.20 & $0.1-5719.80$ \\
DISCERN score & 33.5 & 16 & $16-71$ \\
View ratio & 6 & 6 & $0-19$ \\
VPI score & 1.5 & 2 & $1-4$ \\
\hline
\end{tabular}

GQS: Global Quality Scale; YOCD-SS: YouTube Obsessive-Compulsive DisorderSpecific Score; VPI: Video power index.

was 2.7 minutes in length, with 44 views in 156 days The median length of the videos was 5.78 minutes (min-max: 1.1-70.70; IQR: 8.50), the median number of days since upload of the video was 1014 days (minmax: 7-4985; IQR: 1668). Table 3 shows the descriptive statistics of the 131 videos evaluated.

The median number of views of all of the videos reviewed was 13,745 (min-max: 44-6,815,630; IQR: 50,849 ), the median number of likes was 187 (minmax: 0-180,012; IQR: 888), and the median number of dislikes was 6 (min-max: 0-2507; IQR: 22).

The source of the videos in order of frequency was a psychiatrist/psychologist $(n=63,48.1 \%)$, an educational course $(n=21,16 \%)$, patients/relatives $(n=16,12.2 \%)$, other health professionals $(n=13$, 9.9\%), a documentary $(n=7,5.3 \%)$, a video blogger (vlogger) $(n=4,3.1 \%)$, and other origin $(n=7,5.3 \%)$. Also, it was noted that $9(6.9 \%)$ of the videos were promotional-advertising videos and $11(8.4 \%)$ were created by news agencies.

Calculation of the internal consistency of the quality scores revealed a Cronbach's alpha for the DISCERN instrument of 0.912 and 0.796 for the YOCD-SS.

According to the DISCERN scores, 2 (1.5\%) videos were excellent, $11(8.4 \%)$ were good, 35 (26.7\%) were fair, 45 (34.4\%) were poor, and 38 (29.0\%) were of very poor quality. The items that received a DISCERN score of $\leq 2$ were question 5 $(\mathrm{n}=122,93.1 \%)$, question $4(\mathrm{n}=117,89.3 \%)$, question $8(\mathrm{n}=112,85.4 \%)$, question $11(\mathrm{n}=120,91.6 \%)$, question $12(\mathrm{n}=115,87.8 \%)$, question $9(\mathrm{n}=99$, $75.6 \%)$, and question $16(\mathrm{n}=97,74.1 \%)$. 
Table 4: YouTube Obsessive-Compulsive DisorderSpecific Score of each item of the videos reviewed

\begin{tabular}{rrrrrr}
\multicolumn{2}{c}{ 0 point } & \multicolumn{2}{c}{$\mathbf{1}$ point } & \multicolumn{2}{c}{ 2 points } \\
\hline n & $\%$ & n & $\%$ & n & $\%$
\end{tabular}

\begin{tabular}{lcccccc}
\hline $\begin{array}{c}\text { Diagnosis } \\
\text { Q1 }\end{array}$ & 91 & 69.5 & 27 & 20.6 & 13 & 9.9 \\
Q2 & 103 & 78.6 & 22 & 16.8 & 6 & 4.6 \\
Epidemiology & & & & & & \\
Q3 & 109 & 3.2 & 14 & 10.7 & 2 & 6.1 \\
Q4 & 117 & 89.3 & 4 & 3.1 & 10 & 7.6 \\
Q5 & 96 & 73.3 & 17 & 13.0 & 18 & 13.7 \\
Etiology & & & & & & \\
Q6 & 101 & 77.1 & 23 & 17.6 & 7 & 5.3 \\
Q7 & 109 & 83.2 & 15 & 11.5 & 7 & 5.3 \\
Symptoms & & & & & & \\
Q8 & 33 & 25.2 & 40 & 30.5 & 58 & 44.3 \\
Q9 & 35 & 26.7 & 43 & 32.8 & 53 & 40.5 \\
Q10 & 91 & 69.5 & 20 & 15.3 & 20 & 15.3 \\
Treatment & & & & & & \\
Q11 & 76 & 58.0 & 40 & 30.5 & 15 & 11.5 \\
Q12 & 60 & 45.8 & 38 & 29.0 & 33 & 25.2 \\
Prognosis & & & & & & \\
Q13 & 99 & 75.6 & 24 & 18.3 & 8 & 6.1 \\
\hline
\end{tabular}

Q: Question.

The YOCD-SS scores indicated that $0(0 \%)$ videos were excellent, 6 (4.6\%) were good, 18 (13.7\%) were fair, 45 (34.4\%) were poor, and $62(47.3 \%)$ were very poor. The YOCD-SS scoring is shown in Table 4 . Only 35 (27.5\%) videos received a GQS score of $\geq 3$.

Correlations between video length, like ratio, view ratio, VPI, DISCERN, YOCD-SS, and GQS values are presented in Table 5.

There was no significant difference between the YOCD-SS, GQS, and DISCERN scores of the 2 reviewers (YOCD-SS: $\mathrm{z}$ : $-0.184, \mathrm{p}=0.854$; GQS: $\mathrm{z}$ : $-0.522, \mathrm{p}=0.601$, DISCERN: $\mathrm{z}:-0.163, \mathrm{p}=0.870$ ).

\section{DISCUSSION}

A total of 131 English-language videos related to OCD available on YouTube were examined and the qualitative-quantitative properties of the videos were evaluated. According to the DISCERN, GQS, and YOCD-SS scores, most of the videos were of poor quality. There was a positive correlation between the GQS, DISCERN, and YOCD-SS scores, and the video length. There was also a positive correlation between the video length and the like ratio.

Most previous studies that have assessed the quality of YouTube videos related to medical diseases or conditions have reported that the videos provided insufficient information on the subject $(17,18,21)$. For example, the authors of a study of YouTube videos about plastic surgery found that the content was inadequate and that the quality of the videos was low. They emphasized that users should be aware that the information may not be accurate (22). Our findings similarly indicated that most of the videos provided insufficient information about OCD. When used as a source of information about the disease, it could lead to a false understanding of OCD and could negatively affect the treatment of diagnosed patients and delay the diagnosis and treatment process of patients who have not yet been diagnosed.

Videos about psychiatric disorders and treatments such as schizophrenia (23), bipolar mood disorder (24), electroconvulsive therapy (25), and narcolepsy (26) on YouTube have been evaluated in previous studies. The research examined attitudes toward the disease, the accuracy of the definitions, and usefulness as an educational tool. Szmuda et al. (26) reviewed 80 YouTube videos about narcolepsy. They reported a

Table 5: Correlation of quantitative values of reviewed videos with quality score

\begin{tabular}{lcccccc} 
& $\mathbf{1}$ & $\mathbf{2}$ & $\mathbf{3}$ & $\mathbf{4}$ & $\mathbf{5}$ & $\mathbf{6}$ \\
\hline 1. Length (min) & 1.000 & & & & & \\
2. Like ratio & $0.183^{*}$ & 1.000 & & & & \\
3. View ratio & 0.258 & -0.024 & 1 & & & \\
4. VPI score & $0.266^{* *}$ & 0.011 & $0.998^{* *}$ & 1.000 & & \\
5. DISCERN score & $0.307^{* *}$ & -0.073 & $-0.174^{*}$ & $-0.175^{*}$ & 1.000 & 1.000 \\
6. YOCD-SS & $0.273^{* *}$ & -0.034 & $-0.195^{*}$ & $-0.195^{*}$ & $0.772^{* *}$ & $0.855^{* *}$ \\
7. GQS score & $0.261^{* *}$ & -0.066 & -0.152 & -0.150 & $0.788^{* *}$ & 1.000 \\
\hline
\end{tabular}

*: Correlation is significant at 0.05 (2-tailed); **: Correlation is significant at 0.01 (2-tailed); GQS: Global Quality Scale; YOCD-SS: YouTube Obsessive-Compulsive Disorder-Specific Score; VPI: Video power index. 
mean DISCERN score of $27 \pm 8$ and observed that the quality of the videos was poor.

To the best of our knowledge, there is no previous study of the quality and reliability of videos offered in response to a YouTube search using the keyword obsessive-compulsive disorder. There are, however, 2 studies regarding the quality of OCD-related webbased content. The first evaluated the quality of Dutch websites that discussed OCD, and the quality was generally found to be poor (27). In the second study, Klila et al. (28) found that the content quality of OCD websites in English was relatively good. Our study found that the content of YouTube videos about OCD was poor. The definitions of obsession and compulsion were explained relatively well, however, the definition of functional impairment was found to be weak.

Pharmacological therapy (serotonin reuptake inhibitor and clomipramine) and cognitive-behavioral therapies (exposure, response prevention) are recommended for the treatment of OCD based on multiple randomized controlled trials, meta-analyses, and expert guidelines $(29,30)$.

Our YOCD-SS findings revealed that the video content about pharmacological treatment and psychotherapy received only 2 points $(11.5 \%$ and $25.2 \%$, respectively). Weak or insufficient information about pharmacological OCD treatment in comparison to psychotherapy may increase patients' ambivalence about pharmacological treatment or cause disruptions in existing treatment.

It has been established that longer videos are watched less and receive less attention. We thought that the length of the video might be related to the VPI $(17,21)$. However, our research revealed a positive correlation between video length and the VPI. This may be related to the nature of OCD. Obsessive characteristics in viewers may have contributed to an inclination to watch a video until the end. Similar research of other psychiatric diseases may provide more insight on this issue.

The relationship between video length and video quality has been investigated in previous studies that explored similar concepts in the context of other diseases. It has been reported that longer videos were associated with greater quality (17). We observed a positive correlation between the GQS, DISCERN, and YOCD-SS scores and video length. However, a negative correlation was detected between the GQS, DISCERN, and YOCD-SS scores and the VPI in our study. This suggests that the videos of greatest quality were viewed less frequently.
Our study should be evaluated with some limitations. The YOCD-SS was designed by the researchers using other models previously developed for other diseases and was adapted to include information such as diagnostic and epidemiological treatment method options for OCD. It has a high internal consistency with the GQS and DISCERN. Nonetheless, in future studies, a more structured video evaluation scale confirmed by additional research may be warranted. Furthermore, the information provided in the digital environment and elsewhere is changing rapidly. This study is a cross-sectional evaluation with inherent limitations. Studies using a similar search that track changes could provide better suggestions on the impact of YouTube videos.

\section{CONCLUSION}

The YouTube videos labeled "obsessive-compulsive disorder" we reviewed were often of poor quality and provided incomplete information. Online resources have become a widely used source of information, and therefore, content should be recorded by qualified professionals and objectively present all of the information about treatment options and the psychiatric follow-up process. Since psychiatric symptoms are generally more subjective than somatic disease diagnostic criteria, it is particularly important that the videos are objective in definitions of symptoms and when to consult a psychiatrist. This platform and others have become a significant public resource. However, more videos with high-quality, professional content are needed in order to help provide adequate information to patients and others who need qualified guidance.

\begin{tabular}{|c|c|c|}
\hline \multicolumn{2}{|c|}{ Contribution Categories } & \multirow{2}{*}{$\begin{array}{l}\text { Author Initials } \\
\text { H.K., A.C.K., A.B. }\end{array}$} \\
\hline \multirow{3}{*}{ Category 1} & Concept/Design & \\
\hline & Data acquisition & A.B., A.C.K. \\
\hline & Data analysis/Interpretation & H.K., A.C.K. \\
\hline \multirow{2}{*}{ Category 2} & Drafting manuscript & H.K., A.C.K., A.B. \\
\hline & Critical revision of manuscript & H.K. \\
\hline Category 3 & Final approval and accountability & H.K., A.C.K., A.B. \\
\hline
\end{tabular}

Informed Consent: Participants were instructed on the purpose and design of the study, and the informed consents were obtained.

Peer-review: Externally peer-reviewed.

Conflict of Interest: The authors declare that there was no conflict of interest.

Financial Disclosure: No financial support was granted for this research. 


\section{REFERENCES}

1. Prestin A, Vieux SN, Chou W-YS. Is online health activity alive and well or flatlining? Findings from 10 years of the health information national trends survey. J Health Commun 2015; 20:790-798. [CrossRef]

2. McDaid, D, Park A. Online health: Untangling the web. evidence from the bupa health pulse 2010 international healthcare survey. Bupa Health Pulse survey and results (c) 2010 The British United Provident Association Limited. All other content in this report ( $)$ 2010 LSE Enterprise Limited.London, Bupa. Zugriff am, 16, 2011.

3. Akerkar SM, Kanitkar M, Bichile L. Use of the Internet as a resource of health information by patients: a clinic-based study in the Indian population. J Postgrad Med 2005; 51:116.

4. Lawlor E, Breslin JG, Renwick L, Foley S, Mulkerrin U, Kinsella A, et al. Mental health literacy among Internet users. Early Interv Psychiatry 2008; 2:247-255. [CrossRef]

5. Ma H, Lum C, Dai L, Kwok C, Woo J. Potentially inappropriate medication in elderly patients in outpatient clinics. Asian J Gerontol Geriatr 2008; 3:27-33.

6. YouTube by the numbers: Stats, demographics \& fun facts. https://www.omnicoreagency.com/youtube-statistics/. Accessed March 1, 2021.

7. Bae SS, Baxter S. YouTube videos in the English language as a patient education resource for cataract surgery. Int Ophthalmol 2018; 38:1941-1945. [CrossRef]

8. Szmuda T, Rosvall P, Hetzger TV, Ali S, Słoniewski P. YouTube as a source of patient information for hydrocephalus: a contentquality and optimization analysis. World Neurosurg 2020; 138:e469-e477. [CrossRef]

9. Crino R, Slade T, Andrews G. The changing prevalence and severity of obsessive-compulsive disorder criteria from DSM-III to DSM-IV. Am J Psychiatry 2005; 162:876-882.

10. Ruscio AM, Stein DJ, Chiu WT, Kessler RC. The epidemiology of obsessive-compulsive disorder in the National Comorbidity Survey Replication. Mol Psychiatry 2010; 15:53-63. [CrossRef]

11. Bobes J, González M, Bascaran M, Arango C, Saiz P, Bousono M. Quality of life and disability in patients with obsessive-compulsive disorder. Eur Psychiatry 2001; 16:239-245. [CrossRef]

12. Stein MB, Forde DR, Anderson G, Walker JR. Obsessivecompulsive disorder in the community: an epidemiologic survey with clinical reappraisal. Am J Psychiatry 1997; 154:1120-1126.

13. Torres AR, Prince MJ, Bebbington PE, Bhugra D, Brugha TS, Farrell M, et al. Obsessive-compulsive disorder: prevalence, comorbidity, impact, and help-seeking in the British National Psychiatric Morbidity Survey of 2000. Am J Psychiatry 2006 163:1978-1985. [CrossRef]

14. Khazaal Y, Chatton A, Cochand S, Hoch A, Khankarli MB, Khan $\mathrm{R}$, et al. Internet use by patients with psychiatric disorders in search for general and medical informations. Psychiatr Q 2008; 79:301-309. [CrossRef]

15. Haviland MG, Pincus HA, Dial TH. Type of illness and use of the Internet for health information. Psychiatr Serv 2003; 54:1198.
16. Li M, Yan S, Yang D, Li B, Cui W. YouTube (at $^{\mathrm{ma}}$ as a source of information on food poisoning. BMC Public Health 2019; 19:952.

17. Celik H, Polat O, Ozcan C, Camur S, Kilinc BE, Uzun M. Assessment of the Quality and Reliability of the Information on Rotator Cuff Repair on YouTube. Orthop Traumatol Surg Res 2020; 106:31-34. [CrossRef]

18. Erdem MN, Karaca S. Evaluating the accuracy and quality of the information in kyphosis videos shared on YouTube. Spine 2018; 43:E1334-E1339. [CrossRef]

19. Charnock D, Shepperd S, Needham G, Gann R. DISCERN: an instrument for judging the quality of written consumer health information on treatment choices J Epidemiol Community Health 1999; 53:105-111. [CrossRef]

20. Bernard A, Langille M, Hughes S, Rose C, Leddin D, Van Zanten SV. A systematic review of patient inflammatory bowel disease information resources on the World Wide Web. Am J Gastroenterol 2007; 102:2070-2077. [CrossRef]

21. Biggs T, Bird J, Harries P, Salib R. YouTube as a source of information on rhinosinusitis: the good, the bad and the ugly. J Laryngol Otol 2013; 127:749-754. [CrossRef]

22. Gray MC, Gemmiti A, Ata A, Jun B, Johnson PK, Ricci JA, et al. Can you trust what you watch? An assessment of the quality of information in aesthetic surgery videos on YouTube. Plast Reconstr Surg 2020; 145:329e-336e. [CrossRef]

23. Nour MM, Nour MH, Tsatalou O-M, Barrera A. Schizophrenia on YouTube. Psychiatr Serv 2017; 68:70-74. [CrossRef]

24. Szmuda T, Rosvall P, Hetzger TV, Ali S, Słoniewski P. YouTube as a source of patient information for hydrocephalus: a contentquality and optimization analysis. World Neurosurg 2020; 138:e469-e477. [CrossRef]

25. Genc ES, Wu HE, Pinjari OF, Fernandez LA, Henchcliffe BE, Husain $M$, Selek S. Image of electroconvulsive therapy in YouTube videos. J ECT 2020; 36:e19-e21. [CrossRef]

26. Szmuda T, Özdemir C, Fedorow K, Ali S, Słoniewski P. YouTube as a source of information for narcolepsy: A content-quality and optimization analysis. J Sleep Res 2021; 30:e13053. [CrossRef]

27. Serdobbel Y, Pieters G, Joos S. De obsessieve compulsieve stoornis en het internet. Een evaluatie van Nederlandstalige websites aan de hand van kwaliteitsindicatoren [Obsessive compulsive disorder and the internet. An evaluation of Dutch-language websites and quality indicators]. Tijdschr Psychiatr 2006; 48:763773. [Dutch]

28. Klila H, Chatton A, Zermatten A, Khan R, Preisig M, Khazaal Y. Quality of Web-based information on obsessive compulsive disorder. Neuropsychiatr Dis Treat 2013; 9:1717-1723. [CrossRef]

29. Bandelow B, Sher L, Bunevicius R, Hollander E, Kasper S, Zohar J, et al; WFSBP Task Force on Mental Disorders in Primary Care; WFSBP Task Force on Anxiety Disorders, OCD and PTSD. Guidelines for the pharmacological treatment of anxiety disorders, obsessive-compulsive disorder and posttraumatic stress disorder in primary care. Int J Psychiatry Clin Pract 2012; 16:77-84. [CrossRef]

30. Lovell K, Bee P. Implementing the NICE OCD/BDD guidelines. Psychol Psychother 2008; 81:365-376. [CrossRef] 